\title{
BMJ Open Quantifying clinic transfers among people living with HIV in the Western Cape, South Africa: a retrospective spatial analysis
}

\author{
Ana Lucia Espinosa Dice, ${ }^{1}$ Angela M Bengtson, ${ }^{2}$ Kevin M Mwenda, ${ }^{3}$ \\ Christopher J Colvin, ${ }^{4,5}$ Mark N Lurie (1) ${ }^{2}$
}

To cite: Espinosa Dice AL, Bengtson AM, Mwenda KM, et al. Quantifying clinic transfers among people living with HIV in the Western Cape, South Africa: a retrospective spatial analysis. BMJ Open 2021;11:e055712. doi:10.1136/ bmjopen-2021-055712

- Prepublication history for this paper is available online. To view these files, please visit the journal online (http://dx.doi. org/10.1136/bmjopen-2021055712).

Received 22 July 2021 Accepted 03 November 2021

Check for updates

(C) Author(s) (or their employer(s)) 2021. Re-use permitted under CC BY-NC. No commercial re-use. See rights and permissions. Published by BMJ.

For numbered affiliations see end of article.

Correspondence to

Dr Mark N Lurie;

mark_lurie@brown.edu

\section{ABSTRACT}

Objectives For persons living with HIV (PLWH) in longterm care, clinic transfers are common and influence sustained engagement in HIV care, as they are associated with significant time out-of-care, low CD4 count, and unsuppressed viral load on re-entry. Despite the geospatial nature of clinic transfers, there exist limited data on the geospatial trends of clinic transfers to guide intervention development. In this study, we investigate the geospatial characteristics and trends of clinic transfers among PLWH on antiretroviral therapy (ART) in the Western Cape Province of South Africa.

Design Retrospective spatial analysis.

Setting PLWH who initiated ART treatment between 2012 and 2016 in South Africa's Western Cape Province were followed from ART initiation to their last visit prior to 2017. Deidentified electronic medical records from all public clinical, pharmacy, and laboratory visits in the Western Cape were linked across space and time using a unique patient identifier number.

Participants 4176 ART initiators in South Africa (68\% women)

Methods We defined a clinic transfer as any switch between health facilities that occurred on different days and measured the distance between facilities using geodesic distance. We constructed network flow maps to evaluate geospatial trends in clinic transfers over time, both for individuals' first transfer and overall.

Results Two-thirds of ART initiators transferred health facilities at least once during follow-up. Median distance between all clinic transfer origins and destinations among participants was $8.6 \mathrm{~km}$. Participant transfers were heavily clustered around Cape Town. There was a positive association between time on ART and clinic transfer distance, both among participants' first transfers and overall.

Conclusion This study is among the first to examine geospatial trends in clinic transfers over time among PLWH. Our results make clear that clinic transfers are common and can cluster in urban areas, necessitating better integrated health information systems and HIV care.

\section{INTRODUCTION}

South Africa is home to the world's largest HIV epidemic, with approximately 7.7 million
Strengths and limitations of this study

- This study is among the first to visualise and quantify spatially the phenomenon of clinic transfers among persons living with HIV, highlighting the unique spatial characteristics of clinic transfers in the Western Cape Province.

- Our dataset contains all public clinical, pharmacy, and laboratory data, linked using a unique patient identifier number for consistent identification of individuals across visits and facilities.

- We analyse clinic transfers across the entire Western Cape Province, providing spatial descriptions of transfers both in localised urban areas and across long distances within the Province.

- A limitation of this study is that our dataset includes all healthcare visits, as we cannot reliably distinguish HIV visits from non-HIV visits or visits at which ART was dispensed versus others.

- Since our study only used data from the Western Cape Province and lacked detailed patient characteristics, we were not able to analyse clinic transfers between provinces in South Africa or infer motives for transfers.

individuals living with HIV. ${ }^{1}$ Despite having the largest treatment programme in the world, in South Africa only $62 \%$ and $54 \%$ of people living with HIV (PLWH) were on treatment and virally suppressed, respectively, in 2018, highlighting the need for efforts to improve retention in care. ${ }^{2}$ For PLWH in long-term care, clinic transfers may be an important factor influencing sustained engagement in HIV care. ${ }^{3-5}$ In South Africa, significant proportions of PLWH deemed to be lost to follow-up (LTFU) actually transferred to another clinic; these estimates range from one-third to nearly twothirds. ${ }^{6}$ However, there is evidence that these transfers affect continuity of care for these patients, as interrupted care is associated with significant gaps in ART access and 
adherence.$^{6-8}$ Namely, patients who transfer within province exhibit substantial time out-of-care, low CD4 cell count, and unsuppressed viral load on re-entry. ${ }^{7}$ Overall, gaps in access to clinical care not only lead to an inefficient use of resources, including inaccurate ART drug forecasting and programme evaluation and underestimated rates of retention, but also negatively impact HIV clinical outcomes. ${ }^{67}$

Despite the geospatial nature of clinic transfers, there exist limited data on the geospatial trends of clinic transfers to guide intervention development. ${ }^{7}$ Spatial analysis can help to identify high-risk areas or health facilities where transfers are most frequent as well as common patterns of movement between these spaces, thereby serving as a functional research tool for intervention development to improve engagement in HIV care. ${ }^{9}$ A strong spatial understanding of clinic transfers will complement our overall understanding of the HIV epidemic in South Africa and inform policy decisions about resource allocation and service delivery and design, including patient care coordination and referral systems.

In addition, there are specific characteristics of South Africa's HIV epidemic that uniquely necessitate this geospatial lens. South Africa exhibits a high rate of population mobility, where working-age adults move frequently for employment, a pattern which inevitably fuels clinic transfers. ${ }^{1011}$ Both in-migrants (new residents) and return migrants (former residents returning) exhibit higher infection and mortality risk from both noncommunicable and infectious diseases. ${ }^{12-14}$ Mortality due to HIV and TB is four times higher among some South African migrant populations than among non-migrants. ${ }^{12}$ Migrants also face barriers to retention in care and are less likely to be retained at each step of the HIV treatment cascade. ${ }^{15}$ Given the associations between continuity of care and positive HIV care outcomes, the clinical impact of transfers may at least partially explain this excess risk among highly mobile populations. A spatial understanding of clinic transfers is thus critical in developing effective interventions to support engagement in care among mobile populations. Ultimately, spatial methods will provide insight into coordination, surveillance, and identification systems that can better identify and address issues of patient mobility.

In this study, we investigate the geospatial characteristics and trends of clinic transfers among 4176 PLWH on ART in the Western Cape Province of South Africa. We use visualisation aids and quantify travel distances in order to offer insights into the geographical distribution and patterns of clinic transfers, allowing for more informed decision-making about how to strengthen retention in care in South Africa.

\section{METHODS}

\section{Study setting and population}

This analytic sample came from a cohort of PLWH who tested positive for HIV in Klipfontein, a health subdistrict of the City of Cape Town metropolitan municipality in the Western Cape. ${ }^{16}$ The Western Cape is one of nine provinces of South Africa and in 2016 had a population of 6.3 million people, four million of whom were located in the City of Cape Town. ${ }^{17}$ HIV prevalence among adults in the Western Cape is $12.6 \%$, which is lower than the national average, though the City of Cape Town experiences a particularly high HIV burden. ${ }^{18}$ Klipfontein is among the top three most impoverished subdistricts of Cape Town, with a large proportion of individuals living in informal settlements, a high burden of HIV, TB, and homicide mortality, and heavy reliance on minibus taxis for transportation to clinics. ${ }^{19}{ }^{20}$ Between 2012 and 2015, PLWH in South Africa with a CD4 count $<350$ cells/ $\mathrm{mm}^{3}$ were eligible to initiate lifelong ART. ${ }^{21}$ South Africa adopted universal ART for all pregnant and breastfeeding women (Option B+) in 2015 and for all PLWH in 2016, regardless of $\mathrm{CD} 4$ count or clinical stage. ${ }^{22} 23$

Participants were included if they tested positive for HIV at any health facility in the health subdistrict during 2012 and 2013, if they were older than 15 at the time of testing, and if they initiated ART during the follow-up period. Using Provincial Health data, we then documented all clinical encounters among this cohort at any public health facility in the Western Cape until the end of 2016. Specifically, we included participant visits from their ART initiation date, as reported in their medical records, through their last healthcare visit prior to 31 December 2016, the last date for which visit data was available.

\section{Data sources}

\section{Clinical}

We accessed deidentified electronic medical records through the Provincial Health Data Centre (PHDC) of the Western Cape Department of Health. This dataset contains information on all public clinical, pharmacy, and laboratory visits from health facilities across the Western Cape. These data were linked by the PHDC using a unique patient identifier number, which permits consistent identification of individuals across visits and facilities within all districts of the Province. This data linkage effort has been described elsewhere. ${ }^{24} 25$ A deterministic linkage algorithm that relies on civil identification numbers as well as text-edit-distance fuzzy comparisons was used to reliably link patients' visits across health facilities and remove duplicate identities. ${ }^{24}$

\section{Spatial}

Of the 302 recorded health facilities visited by participants in the study population, we identified and included 237 unique sites visited by individuals on ART; the rest consisted of duplicate facilities or ones visited prior to ART initiation. We manually geocoded all 237 health facilities using Western Cape Government facility addresses and Google Maps. When addresses were not available on the Western Cape Government website, we relied on Google Maps' open-source search engine to identify facility locations. Two clinics were mapped in-person for 
accuracy. Facility types were likewise obtained on the Western Cape Government website whenever possible and on Google otherwise. Facility types included clinics, hospitals, mobile services, reproductive health facilities/ midwife obstetric units (MOUs), and an 'other' category that was used to group rehabilitation clinics, prisons, non-governmental organizations (NGOs), hospices, and dental clinics. Approximately $91 \%$ of health facilities were geocoded and facility type obtained with Western Cape Government data and the rest with an open-source search engine.

\section{Measures}

Clinical

We defined a clinic transfer as any visit to two health facilities that occurred on different days. When a participant visited multiple health facilities on the same day, we used the visit at the most frequented site over the follow-up period in order to determine clinic transfers. Each unique transfer between two healthcare facilities was counted as a clinic transfer, meaning that multiple transfers between the same two sites were counted individually. Information was not available on whether a transfer was official (ie, the origin clinic formally referred the patient) or unofficial (ie, the patient moved independently without a formal referral or transfer letter).

\section{Spatial}

Each clinic transfer consisted of both an origin and a destination healthcare facility. Across a participant's clinical encounters, a facility could act as both an origin and a destination at different time points, as transfers could occur in which a participant returned to a site previously visited. For ease of interpretation and given South Africa's varied road network, we measured the distance between the origin and destination facilities of a transfer using geodesic distance.

\section{Spatial analysis}

The goal of this spatial analysis was to evaluate geospatial trends in clinic transfers over time among this cohort of ART initiators. To do so, we constructed network flow maps to quantify distance between all clinic transfer origins and destinations using ArcMap's XY to Line tool, which connects two points in space with a geodesic (straight) line. Network flow maps depict movement across time and space, allowing us to visualise all clinic transfers across the Western Cape Province from 2012 to 2016. All spatial visualisations and analyses were conducted using ESRI ArcMap V.10.6. ${ }^{26}$ Descriptive statistics of participant characteristics, travel distance, and other transfer summary measures were calculated using STATA (V.15.1) software. ${ }^{27}$

\section{Patient and public involvement}

Patients or the public were not involved in the design, or conduct, or reporting, or dissemination plans of our research.

\section{RESULTS}

Among 8382 individuals who tested positive for HIV in Klipfontein in 2012 or 2013, 4176 (49.8\%) were on ART treatment at some point between 2012 and 2016 and thus were included in our analytic sample. Most individuals initiated ART during 2012 (1311, or 31.4\%) or 2013 (1771, or $42.4 \%)$. Only 561 (13.4\%), 477 (11.4\%), and 56 (1.3\%) individuals initiated ART in 2014, 2015, and 2016, respectively. The majority of ART initiators were women (67.7\%; table 1). Median age at HIV diagnosis was lower for women than for men (29.0 vs 36.0). Furthermore, at ART initiation, median CD4 count among women was almost 100 cells higher than that of men (272.9 vs 179.7). Median follow-up duration on ART was 31.6 months (36.2 for those tested in 2012 vs 27.9 for those tested in 2013). Only 1912 (56.5\%) of the 3383 individuals who had testing location data available initiated ART at the same facility at which they were tested, including a higher proportion of men and a higher proportion of those tested in 2013. The most common testing sites included large community health clinics and reproductive units, though one hospital also sourced $8 \%$ of tests among this cohort. The most common ART initiation sites included large community health clinics.

Among all ART initiators, the median number of clinic transfers during follow-up was 2 (IQR $0-5$ ), and the median number of transfers per year on ART was 0.98 (IQR 0-2.6). Overall, 33\% of individuals never transferred, $9 \%$ transferred once, $13 \%$ transferred twice, and $10 \%$ transferred 10 or more times during follow-up. Among the $67 \%$ of ART initiators who transferred health facilities at least once during follow-up, women were more likely than men to transfer at least once $(56.6 \%$ of men vs $71.9 \%$ of women). Among the 2797 participants who transferred at least once, a total of 14849 clinic transfers occurred across 237 clinics.

Figure 1 shows a cumulative spatial visualisation of the first clinic transfers that occurred during participants' follow-up during ART treatment from 2012 to 2016 (ie, participants' first transfer following ART initiation, herein referred to as 'first transfers'). This includes one transfer for each of the 2797 participants who transferred at least once during follow-up. Figure 1A shows the Western Cape Province within South Africa at large. Figure 1B shows participant movement across the entire Western Cape Province. Figure 1C-E shows the high concentration of clinic transfers surrounding and within the City of Cape Town metropolitan municipality and the Klipfontein health subdistrict. Out of 2797 first transfers, 2694 (96\%) occurred within the Cape Town municipality. Approximately 1800 first transfers (65\%) originated from Klipfontein, and 20\% occurred entirely (both origin and destination) within Klipfontein. Not all first transfers originated in Klipfontein because participants may have transferred from their initial HIV testing site to a new healthcare facility between HIV testing and ART initiation, a move that falls outside the purview of this analysis about transfers after ART initiation. In figure 1D, hubs of 


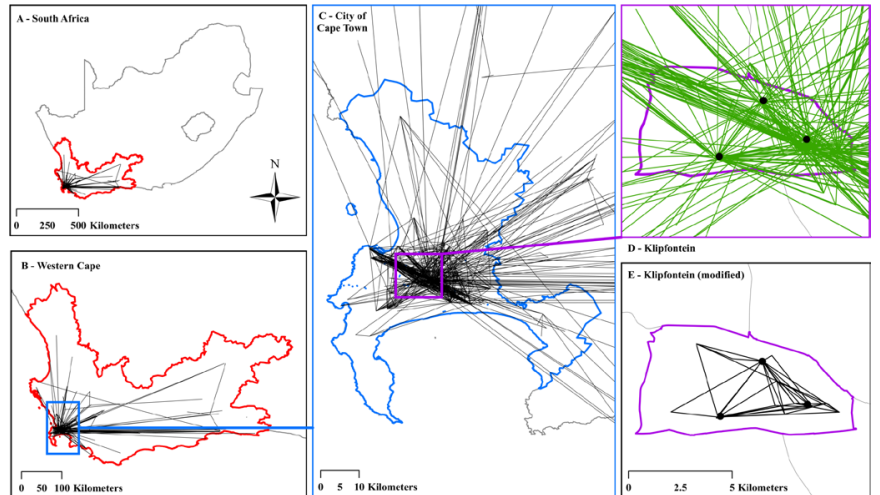

Figure 1 Network flow map of participants' first clinic transfer during ART treatment. Panel (A) contextualises the Western Cape Province (outlined in red) within South Africa. Panel (B) (the Western Cape outlined in red) depicts the first clinic transfer of each of the 2797 individuals who transferred at least once following ART initiation. A black line represents at least one transfer between facility endpoints. Direction of transfer is not specified. Panel (C) zooms into the City of Cape Town metropolitan municipality outlined in blue. Out of 2797 first transfers, 2694 (96\%) occurred within the Cape Town municipality. Panel (D) further zooms into the Klipfontein health subdistrict (outlined in purple) where all participants first tested positive for HIV in 2012 or 2013. A green line depicts a transfer whose origin facility was within Klipfontein (1823 out of 2797 transfers, or $65 \%$ ). The black dots mark specific hubs of participant movement originating from within Klipfontein. Panel (E) removes the traffic across the Klipfontein boundary, depicting only those 567 first clinic transfers $(20 \%)$ that occurred strictly within Klipfontein (outlined in purple).

participant movement within Klipfontein are visible. At the southern centre of Klipfontein, two health facilities account for approximately $13 \%$ of first transfer origins. To the north, one facility accounts for approximately $7 \%$ of first transfer origins. At the eastern tip of Klipfontein, three facilities account for nearly $50 \%$ of first transfer origins. These hubs are made up of large community health centres that offer extensive HIV outreach, testing, and treatment services.

Median distance between all clinic transfer origins and destinations among participants on ART was $8.6 \mathrm{~km}$ (IQR 2.7-12.3; table 2). Only $1.2 \%$ of clinic transfers occurred over a distance greater than $100 \mathrm{~km}$, whereas $36.8 \%$ occurred over a distance less than $5 \mathrm{~km}$. Overall, $14284(96 \%)$ of clinic transfers that occurred after ART initiation were within the Cape Town municipality, with a maximum transfer distance of $52.8 \mathrm{~km}$. More participants transferred facilities within Cape Town, and participants displayed more frequent transfers within Cape Town than beyond. Overall, $24.8 \%$ of clinic transfers occurred within the Klipfontein health subdistrict.

Observable differences in distance between facilities involved in a clinic transfer exist according to time on ART, year of ART initiation, sex, CD4 count at ART initiation, transfer location, and age at time of transfer (table 2). There was a trend towards longer distances 
Table 2 Summary statistics of transfer frequency and distance among those who transferred at least once while on ART

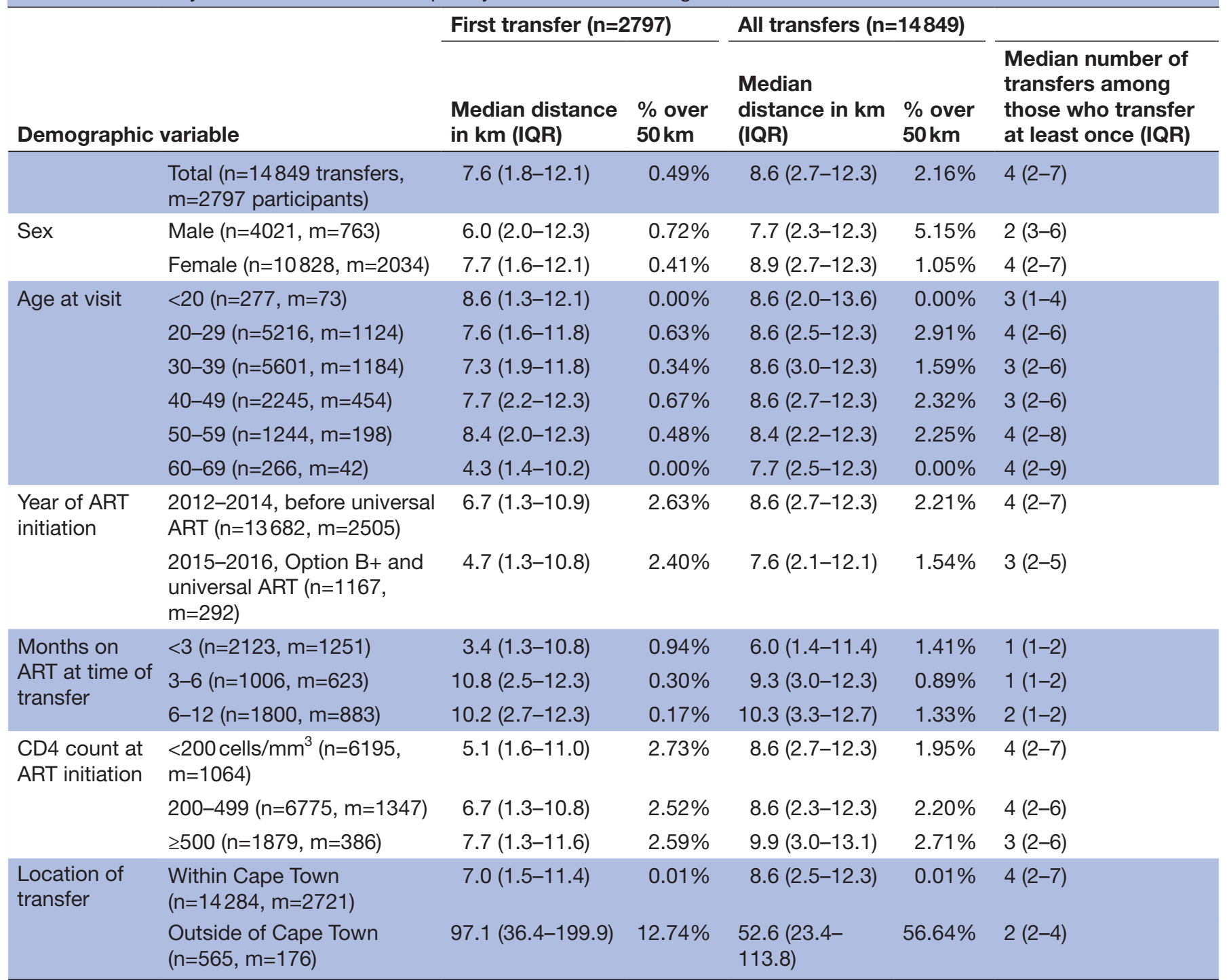

Table 2 shows summary statistics for a variety of transfer distance and frequency measures among the 2797 participants who transferred at least once during their time on ART. For sample sizes, $n$ represents the number of total transfers during the specified time period and by the specified cohort while on ART, and $m$ represents the number of participants in the specified cohort that transferred at least once during the specified time period. The distance $50 \mathrm{~km}$ was chosen in reference to the approximate square root of the square area of the City of Cape Town metropolitan municipality $\left(2444 \mathrm{~km}^{2}\right)$.

between clinic transfers with more time on ART, both among participants' first transfers and overall. Median transfer distance was $6.0 \mathrm{~km}$ among all transfers that occurred within 3 months of ART initiation, $9.3 \mathrm{~km}$ among those between 3 and 6 months of ART initiation, and $10.3 \mathrm{~km}$ among those between 6 months and 1 year of ART initiation. Individuals who initiated ART in 2015 or 2016 tended to travel less far than those who initiated ART between 2012 and 2014 (median 7.6 vs 8.6 $\mathrm{km}$ ). Furthermore, women travelled farther on average than did men, both in their first transfer and across the entirety of their transfers while on ART. Median distance of the first transfer among women was $7.7 \mathrm{~km}$ (IQR 1.6, 12.1) versus 6.0 (IQR 2.0-12.3) among men. Despite this, only $1 \%$ of all transfers among women occurred across a distance of over $50 \mathrm{~km}$, compared with $5 \%$ of all transfers among men. Those with a relatively low CD4 count at ART initiation exhibited substantially lower transfer distances both among their first transfer and overall. On average, individuals aged 60 and above travelled shorter distances than younger individuals, though the small sample size of older adults prevent us from drawing robust conclusions.

Aggregating across all destination facility types, approximately $60 \%$ of all transfers originated at clinics, which generally provide primary care services for chronic diseases, reproductive health, $\mathrm{HIV}$ and $\mathrm{TB}$, and acute conditions (table 3). One-third (33\%) of transfers occurred between two clinic facilities (a clinic as both origin and destination). Approximately 27\% of transfers occurred between a clinic origin and a hospital destination, 
Table 3 Categorising origins and destinations of clinic transfers by facility type

Facility type of destination of clinic transfer

\begin{tabular}{|c|c|c|c|c|c|c|c|}
\hline & & & & & & \multirow{3}{*}{ Row total } \\
\hline & & Clinic & Hospital & $\begin{array}{l}\text { Mobile } \\
\text { services }\end{array}$ & Other & $\begin{array}{l}\text { Reproductive health } \\
\text { facility/MOU }\end{array}$ & \\
\hline \multirow{6}{*}{$\begin{array}{l}\text { Facility type } \\
\text { of origin } \\
\text { of clinic } \\
\text { transfer }\end{array}$} & Clinic & $33.39 \%$ & $27.14 \%$ & $0.01 \%$ & $0.22 \%$ & $1.66 \%$ & \\
\hline & Hospital & $26.61 \%$ & $6.49 \%$ & $0.01 \%$ & $0.11 \%$ & $0.52 \%$ & $33.74 \%$ \\
\hline & Mobile services & $0.07 \%$ & $0.01 \%$ & $0.00 \%$ & $0.00 \%$ & $0.00 \%$ & $0.08 \%$ \\
\hline & Other & $0.22 \%$ & $0.08 \%$ & $0.00 \%$ & $0.07 \%$ & $0.00 \%$ & $0.38 \%$ \\
\hline & $\begin{array}{l}\text { Reproductive } \\
\text { health facility/MOU }\end{array}$ & $2.74 \%$ & $0.63 \%$ & $0.00 \%$ & $0.00 \%$ & $0.02 \%$ & $3.39 \%$ \\
\hline & Column total & $63.03 \%$ & $34.35 \%$ & $0.01 \%$ & $0.40 \%$ & $2.20 \%$ & $100.00 \%$ \\
\hline
\end{tabular}

Table 3 represents a relative frequency matrix of the $n=14849$ clinic transfers that occurred among ART initiators during the period of their ART treatment, categorising transfers according to facility type of the transfer origin and facility type of the transfer destination. Red cells mark a relative frequency value above $1 \%$. Grey cells mark a relative frequency value of $0 \%$.

and an additional $27 \%$ occurred in the reverse direction. Overall, this includes $1856(66 \%)$ individuals who transferred from a hospital and 1866 individuals (67\%) who transferred to a hospital; across all visits (including nontransfers), only $14 \%$ of visits occurred at a hospital. Other significant portions of movement included: $6 \%$ between two hospitals, $3 \%$ between a reproductive health facility/ MOU origin and a clinic destination, and 2\% from a clinic to a reproductive health facility/MOU.

\section{DISCUSSION}

Among over 4000 adults who initiated ART in the Western Cape, South Africa, 67\% transferred at least once during the follow-up period, making clinic transferring a common phenomenon. Clinic transfers occurred across a median distance of $8.6 \mathrm{~km}$. On average, women transferred facilities more frequently and across larger distances; however, a greater proportion of men transferred across a distance of over $50 \mathrm{~km}$. Longer time on ART and higher CD4 count at ART initiation were both positively associated with greater transfer distance. Those who initiated ART in 2015 or 2016 tended to transfer across shorter distances than those who initiated ART between 2012 and 2014.

In this analysis, participant transfers were heavily clustered around the City of Cape Town metropolitan municipality. In addition, one out of every four transfers occurred within Klipfontein itself. Participants exhibited minimal movement across the entire province (only $1 \%$ of transfers occurred over a distance of $100 \mathrm{~km}$ ). It is important to note that since our population was sampled from a cohort of individuals testing positive for HIV at an urban site, our analysis may not be generalisable to rural populations or populations with high rural-to-urban migration. In addition, since our data were confined to the Western Cape Province, we were unable to quantify clinic transfers to other provinces. Given high rates of migration between Cape Town and the Eastern Cape Province, we expect to have missed a significant proportion of transfers. While migration is typically conceived of as long-distance travel, this analysis suggests that patient mobility across short distances within urban areas may account for a large proportion of clinic transfers in some more urban settings. Prior evidence suggests that participants who transfer clinics within province exhibit worse retention and clinical outcomes than individuals who transfer across provinces (likely for relocation). ${ }^{7}$ The dominance of urban transfers-potentially driven by the clinic shopping pattern described by Clouse et al-identified through our geospatial approach further supports the importance of addressing clinic transfers in efforts to improve retention in HIV care.

Our results support the need for strongly integrated health information systems and HIV care and inform where these efforts might most effectively be concentrated geographically. As individuals transfer clinics during longterm HIV care, both within urban hubs and across long distances, health systems must ensure that access to ART and monitoring of clinical outcomes is uninterrupted. South Africa is largely unique within Africa in terms of its utilisation of patient identification numbers that allow for some tracking of individuals in HIV care across space and time. ${ }^{28}$ Specifically, the PHDC's extensive data linkage efforts, ongoing since 2012, benefit not only research and operational purposes, but also clinical care through comprehensive integration of patient data and easier identification of patients in need of intervention. ${ }^{24}$ In the context of HIV, these efforts are critical to ensure uninterrupted HIV care regardless of where a patient chooses to receive care and, concomitantly, to prevent further transmission.

Given the high rates of mobility among the South African population, expansive referral and surveillance systems are crucial in ensuring continuity of care within the large spatial catchment area across which patients transfer clinics. Systems of patient identification across space and time and comprehensive HIV information systems could be foundational to these improved retention and 
surveillance efforts, both within South Africa and elsewhere. ${ }^{29}$ These systems are critical not just among longdistance migrant populations, but also among patients of high mobility within contained areas of urbanisation. Further inquiry into this urban-concentrated movement is key. Additionally, in this cohort, transfer distance increased with time on ART, potentially reflecting the high mobility of the population over time. However, transfers within 3 months of ART initiation, when the risk of LTFU is highest, were most commonly within the same subdistrict. ${ }^{30}$ These results suggest that integrated health systems where patients can access ART across a range of clinics may be particularly important around the time of ART initiation, when patients are evaluating the best option for accessing ART.

Our study also provides important insights into the role of gender, CD4 count, and year of ART initiation on patient movement. Those who initiated ART during 2015 or 2016 - when a test-and-treat approach was first implemented in South Africa through Option B+ and universal ART-exhibited shorter transfer distances compared with those who initiated ART earlier. This pattern is likely explained by the increasing decentralisation of services following universal ART guidelines whereby patients had more options for where to seek care. ${ }^{31}$ Those with CD4 counts $<200$ cells at ART initiation exhibited some of the shortest transfer distances, whereas those with a higher CD4 count travelled relatively far. This latter trend may at least partially explain why patients who initiate ART with a higher CD 4 count are at increased risk of LTFU.$^{32}$ Finally, women transferred more frequently and across larger distances. This gendered pattern of transfer frequency might be driven in part by pregnant women who switch from standard to prenatal care and back, although we did not have data on pregnancy status to verify this hypothesis. The pattern of distance is more surprising, as men largely drive the migration patterns in South Africa. ${ }^{14}$ That being said, women fear inadvertent HIV status disclosure and associated stigma, abandonment, or violence from partners; longer transfer distances among women might be due in part to the desire to hide HIV care from loved ones at a clinic further from home. ${ }^{33}$

This study is among the first to visualise and quantify spatially the phenomenon of clinic transfers among persons living with HIV. This analysis has allowed us to identify the unique spatial catchment area that is required when public health officials call for integrated and continuous HIV care. Our unique dataset contains all public clinical, pharmacy, and laboratory data from this sample of patients within the Western Cape, allowing us to use the plenitude of clinic-based data that exist to map clinic transfers-data that has been shown to successfully capture the broad spatial structures of HIV epidemics. ${ }^{34}$ While other studies offer more insight into patient characteristics and the motives for transfers, these studies often rely on active patient tracing, which is costly and not scalable to a large sample size such as ours. ${ }^{35} 36$ Overall, both kinds of data are critical to deepening our understanding of how to better support individuals who transfer during HIV care.

Various study limitations should be considered. First, we manually geocoded study health facilities using opensource data because of the lack of complete addresses available for these clinics. Thus, it is possible that health facility coordinates are not entirely accurate and that some sites were double-counted. Similarly, our dataset includes all healthcare visits, as we cannot reliably distinguish HIV visits from non-HIV visits. In particular, we do not have sufficient granularity to reliably identify visits at which ART was dispensed (ie, pharmacy pickups). These two limitations could lead to over-estimates of transfer frequency during ART, potentially inflating the observed dominance of urban-concentrated movement. That being said, it is likely that other health visits (eg, emergency room visits, primary care check-ups, dental exams) are much less stigmatised compared with HIV visits and that, as a result, patients seek that care as close to home as possible. Our estimates of HIV care transfer distance are then likely under-estimated. Our exclusion of multiple visits to different facilities on the same day could underestimate clinic transfer frequency, though these instances make up only $4 \%$ of all records.

We were also limited by the available data from the PHDC. In particular, our study would have benefited from more behavioural and demographic information, which was not available. We did not have information on the nature of or motive for a transfer. We also did not have access to participants' home addresses, which may have further clarified the impact of transfers and travel distance on retention in care. We acknowledge that clinic closings, clinic location changes, and participant migration and residential movement could not be explicitly accounted for or determined in this analysis. Due to these data limitations, we cannot infer motives for clinic transfers. Additionally, since our study only used data from the Western Cape Province, we were not able to analyse clinic transfers between provinces in South Africa. Finally, our quantifications of transfer distance do not use road network information, thus overlooking elements such as traffic and time cost in clinic transfers. We opted for a geodesic distance measure because it is difficult to obtain accurate and comprehensive spatial information about South Africa's road networks and minibus taxi routes, the most likely mode of transportation for this cohort. We did not add reference features or basemaps on map figures in order to protect the anonymity of health facilities.

Further research might consider the distinct impact of clinic transfers on HIV care experience and outcomes by gender and by time on ART. We hope to understand what patterns and predictors define the movement of patients who transfer most. With more information about health facilities and about participants' home location, we could also investigate whether clinic transfers are driven by facility size, range of services provided, or distance from patients' residence. While we focus on transfers that occur after ART initiation to address the question of 
retention on ART, we encourage consideration of what patient movement looks like during pre-ART care as well given that a significant proportion of participants did not initiate ART at their testing site. Finally, we might investigate interprovince transfers and the phenomenon of clinic transfers among a migrant labour or otherwise longdistance migrant population. These analytical extensions would provide even more nuance to our spatial visualisation and quantification of clinic transfers among persons living with HIV in the Western Cape, South Africa.

\section{Author affiliations}

${ }^{1}$ Department of Biostatistics, Brown University School of Public Health, Providence, Rhode Island, USA

${ }^{2}$ Department of Epidemiology, Brown University School of Public Health, Providence, Rhode Island, USA

${ }^{3}$ Spatial Structures in the Social Sciences (S4), Population Studies and Training Center (PSTC), Brown University, Providence, Rhode Island, USA

${ }^{4}$ Division of Social and Behavioural Sciences, School of Public Health and Family Medicine, University of Cape Town, Observatory, Western Cape, South Africa ${ }^{5}$ Department of Public Health Sciences, University of Virginia, Charlottesville, Virginia, USA

Contributors ALED contributed to data analysis, data interpretation, figures, literature search, and manuscript writing. AMB contributed to study design, data interpretation, literature search, and manuscript editing. KMM contributed to study design, data interpretation, figures, and manuscript editing. CJC contributed to data collection, study design, and manuscript editing. MNL conceived of the study and contributed to data collection, study design, data interpretation, literature search, and manuscript editing. MNL takes responsibility for the contents of the article.

Funding This work was supported by the National Institute of Mental Health (NIMH) and the South African Medical Research Council (R01 MH106600) as well as the Fogarty International Center at the NIH (D43TW011308-01). Dr. Bengtson was supported through a grant from the National Institute of Mental Health (R00 MH112413). Ms. Espinosa Dice was supported through student research scholarships from the Providence/Boston Center for AIDS Research (P30Al042853) and from the Brown University Undergraduate Teaching and Research Award.

Disclaimer The funding source had no involvement in study design; in the collection, analysis, or interpretation of data; in the writing of the report; or in the decision to submit the paper for publication. The content is solely the responsibility of the authors and does not necessarily represent the official views of the South African Medical Research Council, the National Institute of Mental Health, the Center for AIDS Research, or Brown University.

Competing interests None declared.

Patient and public involvement Patients and/or the public were not involved in the design, or conduct, or reporting, or dissemination plans of this research.

Patient consent for publication Not applicable.

Ethics approval Ethical approval and a waiver of informed consent for the use of deidentified routine data were provided by the University of Cape Town (Protocol number 320/2015).

Provenance and peer review Not commissioned; externally peer reviewed.

Data availability statement No data are available.

Open access This is an open access article distributed in accordance with the Creative Commons Attribution Non Commercial (CC BY-NC 4.0) license, which permits others to distribute, remix, adapt, build upon this work non-commercially, and license their derivative works on different terms, provided the original work is properly cited, appropriate credit is given, any changes made indicated, and the use is non-commercial. See: http://creativecommons.org/licenses/by-nc/4.0/.

\section{ORCID iD}

Mark N Lurie http://orcid.org/0000-0002-3153-1715
REFERENCES

1 UNAIDS. South Africa: overview. Available: https://www.unaids.org/ en/regionscountries/countries/southafrica

2 WHO. Global health sector response to HIV 2000-2015: focus on innovations in Africa, 2015

3 Wilkinson LS, Skordis-Worrall J, Ajose O, et al. Self-transfer and mortality amongst adults lost to follow-up in ART programmes in low- and middle-income countries: systematic review and metaanalysis. Trop Med Int Health 2015;20:365-79.

4 Fox MP, Bor J, Brennan AT, et al. Estimating retention in HIV care accounting for patient transfers: a national laboratory cohort study in South Africa. PLoS Med 2018;15:e1002589.

5 Haas AD, Zaniewski E, Anderegg N, et al. Retention and mortality on antiretroviral therapy in sub-Saharan Africa: collaborative analyses of HIV treatment programmes. J Int AIDS Soc 2018;21:e25084.

6 Tweya $\mathrm{H}$, Feldacker $\mathrm{C}$, Estill J, et al. Are they really lost? "true" status and reasons for treatment discontinuation among HIV infected patients on antiretroviral therapy considered lost to follow up in Urban Malawi. PLoS One 2013;8:e75761.

7 Clouse K, Vermund SH, Maskew M, et al. Mobility and clinic switching among postpartum women considered lost to HIV care in South Africa. J Acquir Immune Defic Syndr 2017;74:383-9.

8 Mutevedzi PC, Lessells RJ, Newell M-L. Disengagement from care in a decentralised primary health care antiretroviral treatment programme: cohort study in rural South Africa. Trop Med Int Health 2013;18:934-41.

9 Boyda DC, Holzman SB, Berman A, et al. Geographic information systems, spatial analysis, and HIV in Africa: a scoping review. PLoS One 2019;14:e0216388.

10 International Organization for Migration. Southern Africa: the regional migration context. Available: https://www.iom.int/southern-africa

11 Collinson MA, White MJ, Bocquier P, et al. Migration and the epidemiological transition: insights from the Agincourt sub-district of northeast South Africa. Glob Health Action 2014;7:23514.

12 Ginsburg C, Bocquier P, Béguy D, et al. Association between internal migration and epidemic dynamics: an analysis of cause-specific mortality in Kenya and South Africa using health and demographic surveillance data. BMC Public Health 2018;18:918.

13 Abubakar I, Aldridge RW, Devakumar D, et al. The UCL-Lancet Commission on migration and health: the health of a world on the move. The Lancet 2018;392:2606-54.

14 Lurie M, Williams B, Zuma K. The impact of migration on HIV-1 transmission: a study of migrant and non-migrant men, and their partners. Sex Transm Dis 2003;40:149-56.

15 Larmarange J, Diallo MH, McGrath N, et al. The impact of population dynamics on the population HIV care cascade: results from the ANRS 12249 treatment as prevention trial in rural KwaZulu-Natal (South Africa). J Int AIDS Soc 2018;21 Suppl 4:e25128.

16 Lurie MN, Kirwa K, Callaway J, et al. Quantifying the HIV treatment cascade in a South African health sub-district by gender: retrospective cohort study. Trop Med Int Health 2020;25:186-92.

17 Ngyende A, Khoza C. Community survey 2016 provinces at a glance, 2016. Statistics South Africa

18 Human Sciences Research Council (HSRC). South African national HIV prevalence, incidence, behaviour and communication survey 2017.

19 Groenewald P, Neethling I, Evans J, et al. Mortality trends in the city of Cape town between 2001 and 2013: reducing inequities in health. S Afr Med J 2017;107:1091-8.

20 Mumm R, Diaz-Monsalve S, Hänselmann E, et al. Exploring urban health in Cape Town, South Africa: an interdisciplinary analysis of secondary data. Pathog Glob Health 2017;111:7-22.

21 Meintjes G, Maartens G, Boulle A. Guidelines for antiretroviral therapy in adults by the southern African HIV clinicians Society. South African J HIV Med 2012;13:114-33.

22 Meintjes G, Black J, Conradie F, et al. Southern African HIV Clinicians Society adult antiretroviral therapy guidelines: update on when to initiate antiretroviral therapy. South Afr J HIV Med 2015;16:428.

23 Department of Health SA. National consolidated guidelines for the prevention of mother-to-child transmission of HIV (PMTCT) and the management of HIV in children, adolescents and adults. Pretoria, South Africa: Department of Health, 2015.

24 Boulle A, Heekes A, Tiffin N, et al. Data centre profile: the provincial health data centre of the Western Cape Province, South Africa. Int J Popul Data Sci 2019;4:1143.

25 Bengtson AM, Colvin C, Kirwa K, et al. Estimating retention in HIV care accounting for clinic transfers using electronic medical records: evidence from a large antiretroviral treatment programme in the Western Cape, South Africa. Trop Med Int Health 2020;25:936-43.

26 ESRI. ArcGIS desktop release 10.6: environmental systems research Institute, 2019 
27 StataCorp. Stata statistical software, 2017. StataCorp LLC

28 Mehta U, Heekes A, Kalk E, et al. Assessing the value of Western Cape provincial government health administrative data and electronic pharmacy records in ascertaining medicine use during pregnancy. $S$ Afr Med J 2018;108:439.

29 Rice B, Boulle A, Baral S, et al. Strengthening routine data systems to track the HIV epidemic and guide the response in Sub-Saharan Africa. JMIR Public Health Surveill 2018;4:e36.

30 Boeke CE, Nabitaka V, Rowan A, et al. Assessing linkage to and retention in care among HIV patients in Uganda and identifying opportunities for health systems strengthening: a descriptive study. BMC Infect Dis 2018;18:138.

31 Myburgh H, Reynolds L, Hoddinott G, et al. Implementing 'universal' access to antiretroviral treatment in South Africa: a scoping review on research priorities. Health Policy Plan 2021;36:923-38.

32 Grimsrud A, Cornell M, Schomaker M, et al. CD4 count at antiretroviral therapy initiation and the risk of loss to follow-up: results from a multicentre cohort study. J Epidemiol Community Health 2016;70:549-55.

33 Bengtson AM, Go V, Kumwenda W, et al. "A way of escaping": a qualitative study exploring reasons for clinic transferring and its impact on engagement in care among women in option B. AIDS Care 2020;32:72-5.

34 Cuadros DF, Sartorius B, Hall C, et al. Capturing the spatial variability of HIV epidemics in South Africa and Tanzania using routine healthcare facility data. Int $J$ Health Geogr 2018;17:27.

35 Yu JK-L, Tok T-S, Tsai J-J, et al. What happens to patients on antiretroviral therapy who transfer out to another facility? PLoS One 2008;3:e2065.

36 Sikombe K, Mody A, Kadota J, et al. Understanding patient transfers across multiple clinics in Zambia among HIV infected adults. PLoS One 2020;15:e0241477. 